\title{
Globoid surface shaped with turning and envelope method
}

\author{
Rafat Gołębski ${ }^{1}$, Piotr Boral $^{1,}{ }^{*}$ \\ ${ }^{1}$ Czestochowa University of Technology, Institute of Mechanical Technologies, Al. Armii Krajowej \\ 21, 42-201 Czestochowa, Poland
}

\begin{abstract}
In industrial practice, cylindrical worm gears are most often used due to their feasibility. In the literature, much attention has been paid to determining the helical surface of worms of such transmissions. Globoidal worm gearboxes are much less frequently used due to technological difficulties. Come on, the globoidal worm ensures simultaneous cooperation of a larger number of teeth and the location of the teeth contact line is more favorable. Therefore, the following is an analytical method for the description of a globoid surface by turning and by a finger milling method.
\end{abstract}

Keywords: globoid worm, helical surface.

\section{Introduction}

Globoid worms can be shaped with a knife by the turning profiling method. Machining by turning is little efficient, and taking into account shaping the convolution surface over the full profile height in the finishing pass, the surface quality parameters and machining accuracy may be low. Worms can be turned with a single knife, but may also be machined by the turning hobbing method using a Fellows knife.

Just like cylindrical worms or wormwheels $[1,2]$, globoid worms can also be machined the hobbing method with a finger mill $[3,4,5]$. Generally, this is a conical finger mill with a rectilinear profile in the axial section of the tool action surface. Hobbing machining is more efficient and more accurate [6]. However, it is more difficult due to the need for ensuring a high rotary speed of the tool with its simultaneous rotation around the theoretical axis of the wormwheel mating with the wormwheel worm. To accomplish such machining on a modern multi-axial, multi-purpose $\mathrm{CNC}$ machine tool, the machine tool must have 5 controlled axes, including the axis of rotation of the workpiece spindle (C) and the possibility of infinitely changing the angle of twist of the tool spindle axis (B). So, in the case of a 5-axis $\mathrm{CNC}$ machine tool with a tilting-rotary table this may be hard to accomplish.

Considering the fact that wormwheel surface should theoretically be machined with a globoid hob, that is a mill with an action surface consistent with the surface of the worm mating with that wormwheel, in the constructional worm gear it is not indifferent which worm surface to select for the globoid hob technology [7,8]. The hob is a tool that is difficult in terms of geometry and technology and, as a rule, differences resulting from the surfaces

\footnotetext{
* Corresponding author: piotrek@itm.pcz.czest.pl

Reviewers: Piotr Krawiec, Ján Vavro Jr.
} 
of action of the hob and the worm are practically impossible to eliminate. Therefore, when machining a worm it should be considered which mill will be more accurate and also easier and cheaper to make - that built based on the turned worm, or that built based on the worm machined by the hobbing method. The wormwheel can also be cut using a special head with a single knife, which is undoubtedly a cheaper solution, compared to machining by milling. But, obviously, such machining will be less efficient and less accurate. Apart from that, the machining kinematics is, anyway, identical to that in hobbing (a knife in a special head imitates one tooth of the hob) [9].

At the same time, the above discussion implies that the wormwheel tooth surface can be determined as the worm surface envelope, on assumption that the hob action surface is identical, which is rather unlikely in practice (of course, a single knife with the required profile corresponding to the worm profile is much easier to make) $[10,11]$.

Another conclusion from this discussion is that the accurate execution of the globoid worm gear is very difficult (practically, rather impossible). We mean gears, where the mating surfaces of the toothing (of the worm and the wormwheel) are, on assumption, mutually enveloping (coupled) [12].

\section{Worm surface shaped by turning machining}

The machining of the globoid worm by turning, due to machining kinematics and the use of special tool heads, is difficult to accomplish. An unquestionable advantage of turning is the fact that a ruled machined surface is obtained at the rectilinear cutting edge of the tool blade, whose accuracy is easy to check.

Relationships describing the worm surface do not require the envelope condition to be formulated and solved, as is the case in hobbing, which, however, does not alter the fact that the globoid hob for wormwheel machining will be geometrically and technologically very complex. Nevertheless, this is the classing (basic) [1] type of globoid worm gear.

\subsection{Tool profile and setting}

The profile of the tool cutting edge can be described with the following equation - Figure 1

$$
\underset{z}{\boldsymbol{x}}(u)=\left[\sqrt{\left(r_{p 2}\right)^{2}-s^{2}}+u, \quad 0, \quad s+u \operatorname{tg} \alpha_{z}\right]^{T}
$$

where: $s$ - blade half-width at the pitch height, $r_{p 2}$ - radius of the wormwheel pitch diameter in the middle section, $\alpha_{z}$ - tool profile angle, $u$ - linear tool profile parameter. The profile angle can be determined from the relationship - Figure 1

$$
\operatorname{tg} \alpha_{z}-\frac{r_{0}-\frac{s}{\cos \alpha_{z}}}{\sqrt{\left(r_{p 2}\right)^{2}-\left(r_{9}\right)^{2}}}=0,
$$

where: $r_{0}$ - base circle radius. 


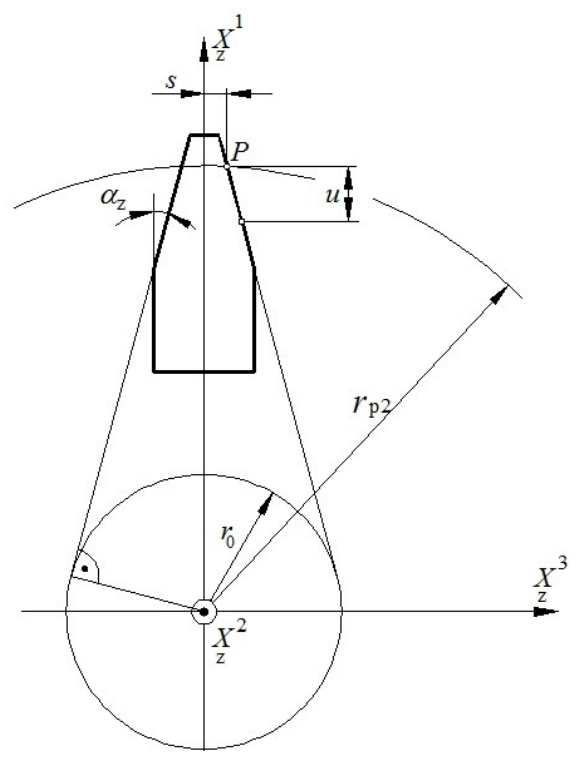

Fig. 1. Tool profile

The profile circle is important, as it defines the maximum worm length, over with convolutions may be cut. Should the worm be cut over a length greater than the diameter of this circle, the globoid worm gear could not be assembled (Fig. 2).

\subsection{The surface of the worm machined by turning}

The surface of a worm convolution in the conventional globoid worm gear [1] is shaped with a straight line (knife cutting edge) rotating in the worm axial plane around the wormwheel axis and simultaneously with this plane around the worm axis, so that the ratio of both rotations is constant - Figure 2 .

The worm and tool rotation angles are related with the relationship that results from the transmission ration

$$
\varphi_{2}\left(\varphi_{1}\right)=\varphi_{1} \frac{z_{1}}{z_{2}}=i \varphi_{1}
$$

where: $z_{1}$ - number of worm convolutions, $z_{2}$ - number of wormwheel teeth, $\varphi_{1}-$ worm rotation angle (tool surface angular parameter), $\varphi_{2}$ - rotation angle of the wormwheel (or the tool), $i$ - transmission ratio.

As follows from relationship (3), the transmission ratio is constant. Therefore, taking into account the tool setting and the relative tool and worm motion, the worm surface can be described with the equation - Figure 2

$$
\boldsymbol{x}\left(u, \varphi_{1}\right)=\left[\begin{array}{lll}
x^{1}, & x^{2}, & x^{3}
\end{array}\right]^{T}=\left[3, \varphi_{1}\right]\left(\left[\begin{array}{lll}
2, & -\varphi_{2}
\end{array}\right] \underset{z}{\boldsymbol{x}}+\left[\begin{array}{lll}
-a & 0, & 0
\end{array}\right]^{T}\right)
$$

Whereas, the distance of the gear link axes is

$$
a=r_{p 1}+r_{p 2}
$$


where: $r_{p 1}$-the smallest worm pitch radius, $a$-distance of the worm and wormwheel axes.

Considering relationship (3), the equation of the worm surface (worm convolution flanks) can be written as

$$
\boldsymbol{x}\left(u, \varphi_{1}\right)=\left[\begin{array}{lll}
x^{1}, & x^{2}, & x^{3}
\end{array}\right]^{T}=\left[\begin{array}{ll}
3, & \varphi_{1}
\end{array}\right]\left(\left[\begin{array}{ll}
2, & -i \varphi_{1}
\end{array}\right] \underset{z}{\boldsymbol{x}}+\left[\begin{array}{lll}
-a, & 0, & 0
\end{array}\right]^{T}\right)
$$

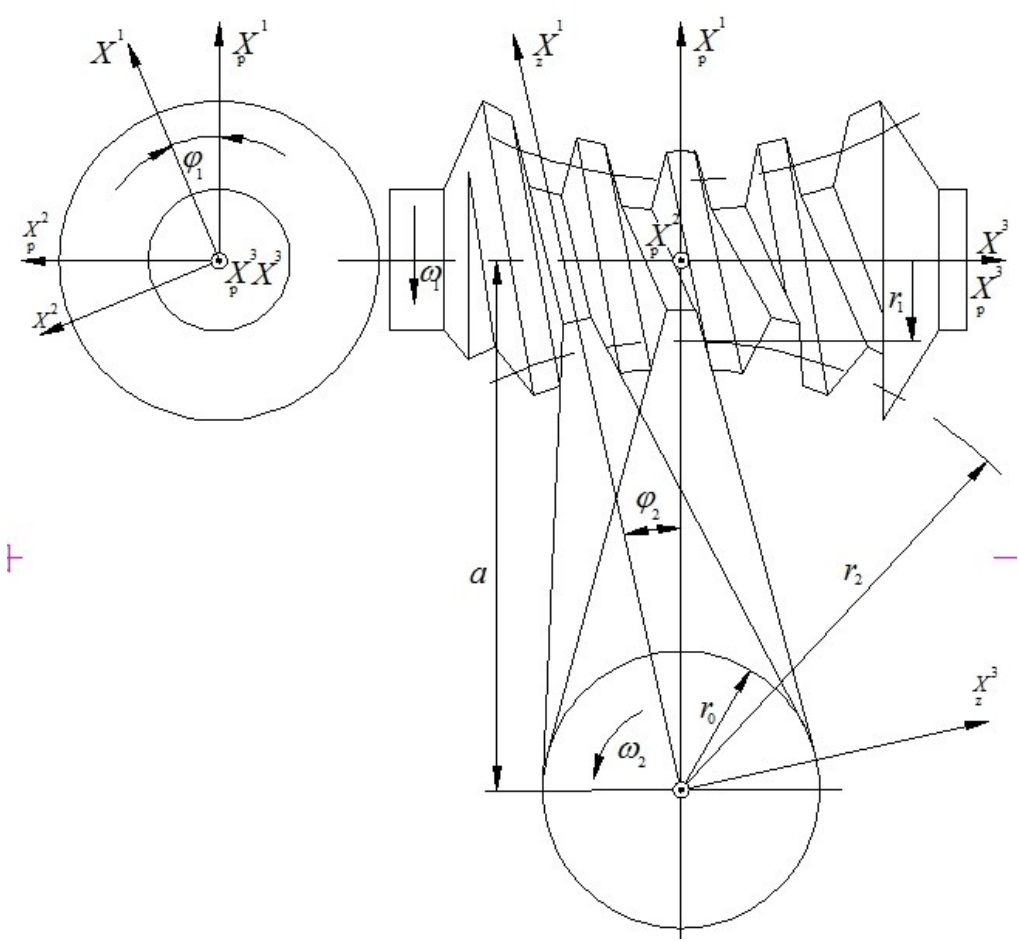

Fig. 2. The tool setting and the kinematics of globoid worm gear machining by turning

The worm axial profile can be determined from the equation

$$
x^{2}\left(u, \varphi_{1}\right)=0
$$

From this equation, for the preset values of parameter $u$, its corresponding values of parameter $\varphi_{1}$ can be calculated, and then the remaining coordinates $\left(x^{1}, x^{3}\right)$ of the worm axial profile can be determined from equation (4). However, in order to determine the profile in the same axial plane for successive "teeth", $\varphi_{1}$

$$
\varphi_{1}=\varphi_{10} \pm 2 k \pi \quad k= \pm 0,1,2, \ldots
$$

where: $\varphi_{10}$ - angle of cutting plane position; $k$ - denotes the „tooth”.

It can be assumed $\varphi_{10}=0$ (it is convenient to do so), although it may be an arbitrary value in the range $\langle 0,2 \pi\rangle$. If we take into consideration a worm with one convolution and cut it with the axial plane, then we will see "subsequent teeth" (formed as a result of intersection of that convolution with this plane) in that section. In the case of a multi-convolution worm, the surfaces of subsequent convolutions are identical, except that rotated relative to one 
another around the worm axis by an angle resulting from the number of convolutions (so, it is sufficient to examine the profile of a single convolution).

For further discussion it is necessary to determine the versors (unit vectors) normal to the worm surface, which can be expressed with the relationship:

$$
\boldsymbol{n}=\frac{\frac{\partial \boldsymbol{x}}{\partial u} \times \frac{\partial \boldsymbol{x}}{\partial \varphi_{1}}}{\left|\frac{\partial \boldsymbol{x}}{\partial u} \times \frac{\partial \boldsymbol{x}}{\partial \varphi_{1}}\right|}
$$

With a fixed value of parameter $\varphi_{10}$, these will be normal to the points of the axial profile defined by the value of that angle (8). As appears from the machining method, the worm profile is constant (is rectilinear, only it rotates and moves correspondingly along the worm axis - Fig. 2). The component vectors of equation (9) are as follows:

$$
\begin{aligned}
& \frac{\partial \boldsymbol{x}}{\partial u}=\left[3, \quad \varphi_{1}\right]\left[2,-i \varphi_{1}\right] \frac{\partial \boldsymbol{x}}{\partial u}
\end{aligned}
$$

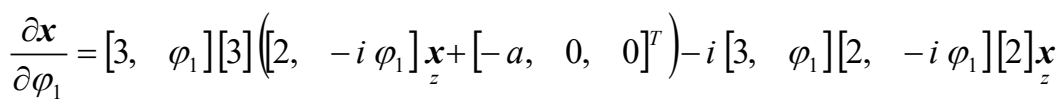

At any point A (in the worm axial plane perpendicular to the wormwheel axis) on the worm helix, the resultant velocity on the pitch surface must be tangential to that helix - Figure 3, or [2]

$$
\operatorname{tg} \gamma=i \frac{r_{p 2}}{r_{1}}=i \frac{r_{p 2}}{a-\sqrt{\left(r_{p 2}\right)^{2}-e^{2}}}
$$

where: $e$ - the distance of the point under consideration on the pitch surface of the worm from its centre.

Whereas:

$$
r_{1}=a-\sqrt{\left(r_{p 2}\right)^{2}-e^{2}}
$$

As follows from the relationship (12), the angle of inclination of the globoid worm convolution helix is variable over the worm length and attains the greatest value in the worm centre (for $e=0$ ). But this angle is also variable across the profile height, because for points not lying on the wormwheel pitch diameter, correspondingly varying radius values can be assumed in this formula. 


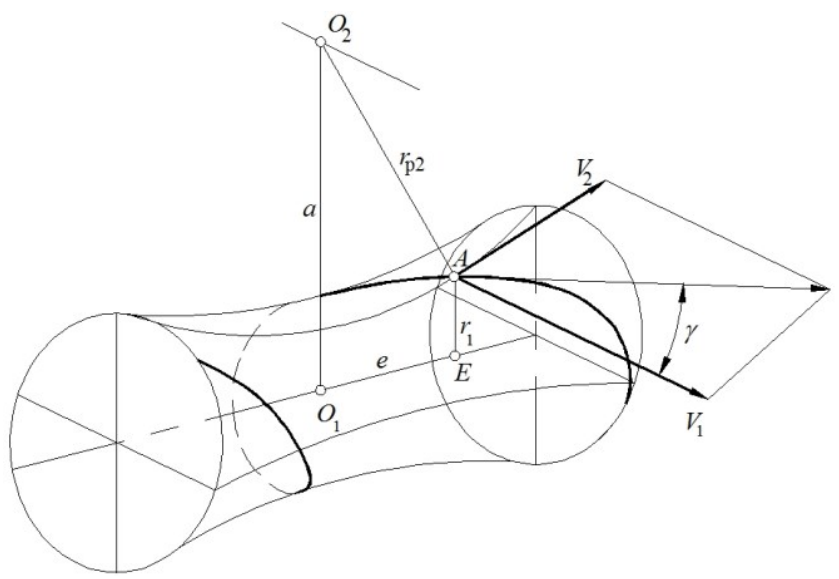

Fig. 3. Setting the lead angle on the pitch surface of the globoid worm

Therefore, in a general case of a point that does not lie on the pitch diameter and does not lie in the worm axial plane perpendicular to the wormwheel axis - Figure 4, the convolution lead angle will be defined by the relationship

$$
\operatorname{tg} \gamma=i \frac{r_{2}}{r_{1}}=i \frac{r_{2}}{a-\sqrt{\left(r_{2}\right)^{2}-e^{2}}}
$$

Whereas:

$$
r_{1}=\frac{a-\sqrt{\left(r_{p 2}\right)^{2}-e^{2}}}{\cos \varepsilon}
$$

where: $r_{2}$ - the radius of the position of the point under consideration, as measured from the wormwheel axis; $\varepsilon$ - the parameter of point position in the plane perpendicular to the worm axis, as defined relative to the interval of the smallest interaxial distance.

This means that relationships (12) and (13) are a special case of relationships (14) and (15).

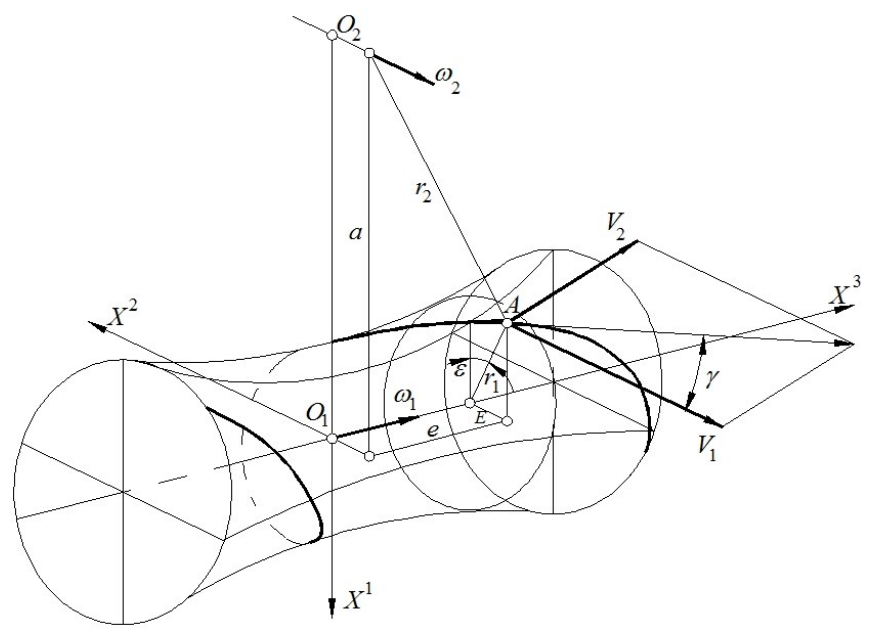

Fig. 4. Setting the lead angle and the normal to the surface of the globoid worm [2] 


\section{Worm surface shaped by the hobbing method}

In the case at hand, the tool is a conical finger mill with a rectilinear axial profile of the tool action surface. Such a tool is geometrically simple and cheap. In the case of tools of small overall dimensions, the tool can be made of sintered carbides as solid, which offers the possibility of dry machining and machining of toughened materials, as well as the possibility of machining with high cutting parameters (compared to a tool of high-speed steel).

\subsection{Tool action surface}

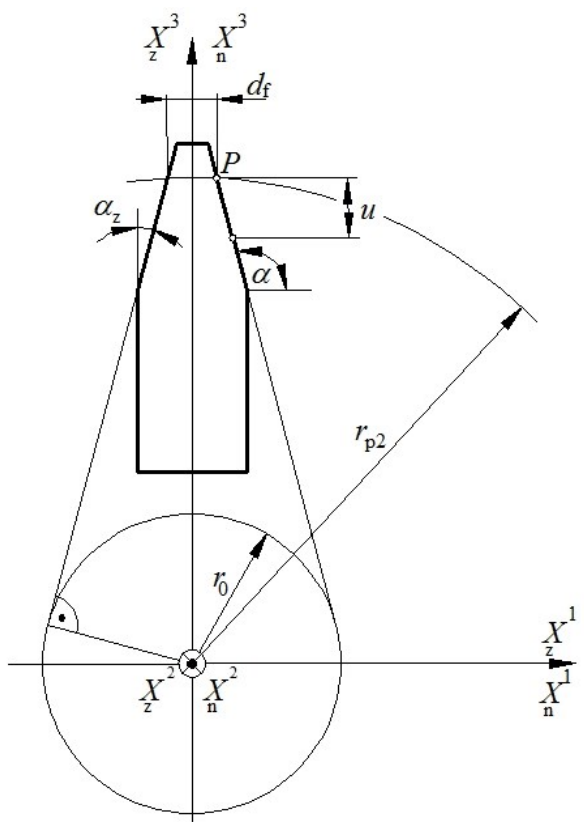

Fig. 5. The axial profile of the finger-type tool

Point $\mathrm{P}$ of the tool axial profile corresponds to the point on the worm pitch diameter. The rectilinear profile of the tool will be described by the equation - Figure 5 .

$$
\underset{z}{\boldsymbol{x}}(u)=\left[ \pm \frac{d_{f}}{2}+u \operatorname{tg} \alpha_{z}, 0, r_{p 2}+u\right]^{T}
$$

where: \pm - for the right-hand and left-hand profile, respectively; $d_{f}-$ analytical tool diameter; $\alpha_{Z}$ - tool axial surface semicircle; $u$ - axial profile parameter.

Whereas, the analytical tool axial profile angle $\alpha$ for the right-hand and left-hand profile side, respectively, is equal to

$$
\alpha=\frac{\pi}{2} \pm \alpha_{Z}
$$

where: \pm - respectively, for the right-hand and left-hand profile side.

The action surface of the tool forms by the rotation of its axial profile around the axis of rotation, as shown in Figure 6. 


$$
\underset{N}{\boldsymbol{x}}(u, \varphi)=[3,-\varphi] \underset{z}{\boldsymbol{x}}(u)
$$

where: $\varphi$ - the parameter of the tool action surface (the angle of axial profile rotation around the $X_{N}^{3}$ axis of tool rotation).

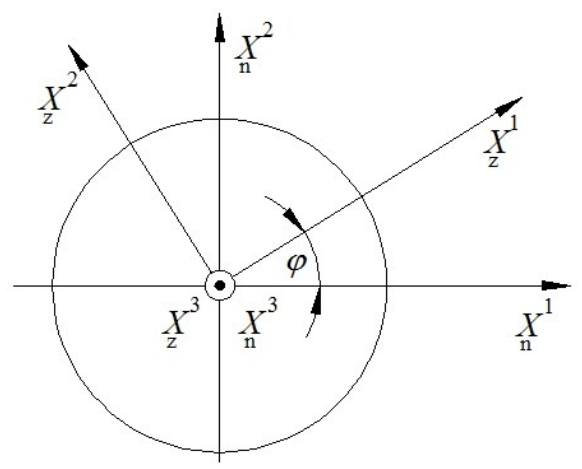

Fig. 6. Schematic diagram of transition from the coordination system of the axial profile $\{z\}$ to that of the tool $\{\mathrm{N}\}$

\section{Worm surface}

For the determination of the worm surface, the tool setting and the machining kinematics should be taken into account, and the family of tool action surfaces needs to be described in the worm's system. Then, the worm surface must be determined from the envelope condition.

\subsection{Tool setting and the relative motion of turning of the tool and the worm being machined}

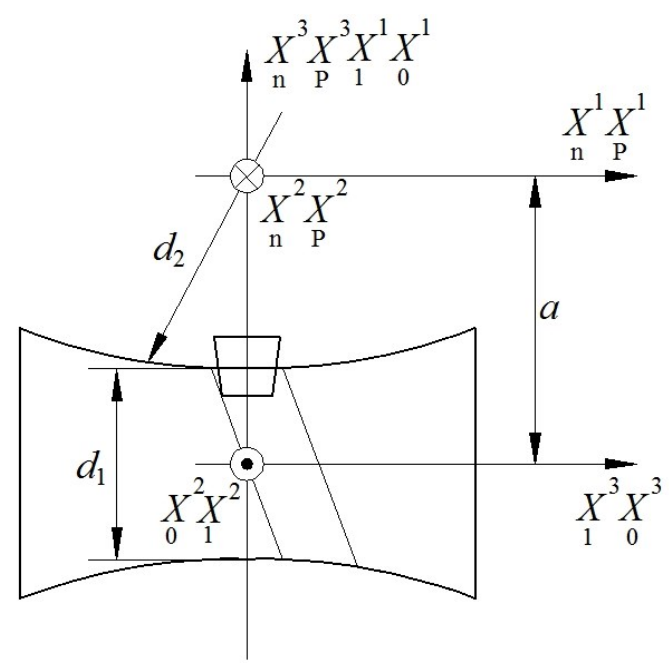

Fig. 7. Schematic diagram of tool and worm setting (in the zero position) 


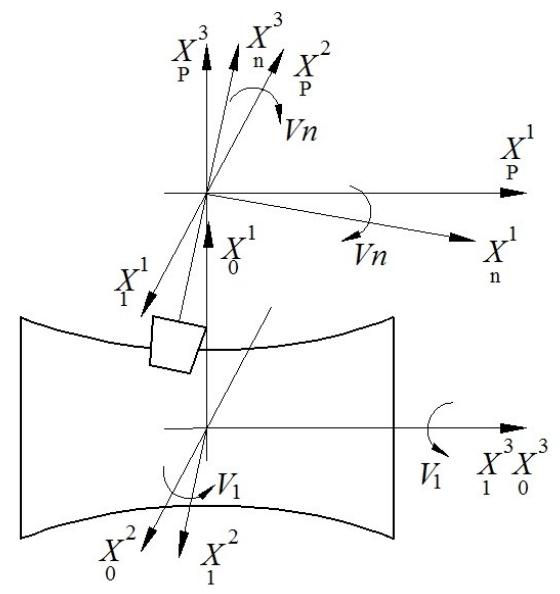

Fig. 8. Schematic diagram of tool and worm turning

Describe the transition for the $\{\mathrm{N}\}$ system of the tool to the $\{1\}$ system of the worm, while considering its setting (Fig. 7) and machining kinematics (Fig. 8), that is, consider the following, in succession:

a) transition from the $\{\mathrm{N}\}$ system of the tool to the $\{\mathrm{p}\}$ auxiliary (fixed) system that, at the initial moment $(\mathrm{vN}=0)$, coincided with the tool's system

$$
\underset{p}{\boldsymbol{x}}=\left[2,-v_{N}\right] \underset{N}{\boldsymbol{x}}
$$

where: $v_{N}-$ the parameter of tool rotation around the axis of rotation of the theoretical wormwheel;

b) transition from the $\{p\}$ auxiliary system of the tool to the $\{0\}$ auxiliary (fixed, initial) system of the worm

$$
\underset{o}{\boldsymbol{x}}=\left[2, \frac{\pi}{2}\right][1, \quad \pi]\left(\underset{p}{\boldsymbol{x}}+[0,0, \quad a]^{T}\right)
$$

where: $a$ - the distance of the axes of rotation of the worm and the wormwheel.

c) transition from the $\{0\}$ reference system of the worm to the $\{1\}$ movable system rigidly coupled with the worm

$$
\underset{1}{\boldsymbol{x}}=\left[3, v_{1}\right] \underset{o}{\boldsymbol{x}}
$$

where: $v_{1}$ - worm rotation angle.

Therefore, the equation of the family of tool action surfaces in the $\{1\}$ worm system is as follows

$$
\underset{1}{\boldsymbol{x}}\left(u, \varphi, v_{1}\right)=\left[3, v_{1}\right]\left[2, \frac{\pi}{2}\right]\left[\begin{array}{ll}
1, & \pi
\end{array}\right]\left(\left[\begin{array}{ll}
2, & -v_{N}
\end{array}\right] \underset{N}{\boldsymbol{x}}+\left[\begin{array}{lll}
0, & 0, & a
\end{array}\right]^{T}\right)
$$

where the worm and tool rotation angles are related with the function of worm gear (worm) transmission ratio 


$$
v_{N}=v_{1} \frac{z_{1}}{z_{N}}
$$

where: $z_{1}, \quad z_{N}$ - the number of worm and wormwheel teeth.

So, the equation of the family of surfaces (22) will assume the form

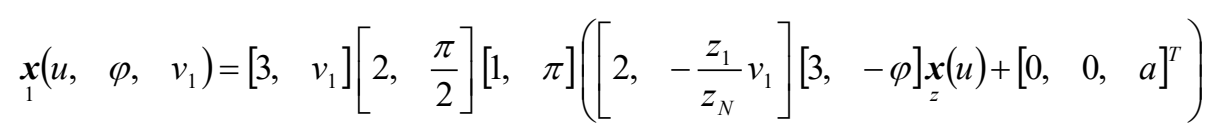

\subsection{The envelope condition for shaping the worm helical surface}

To describe the worm surface, to equation (24) for the family of tool action surfaces, the envelope conditions needs to be added, which can be generally written as

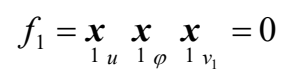

where:

$$
\begin{aligned}
& \underset{1 u}{\boldsymbol{x}}=\frac{\partial \boldsymbol{x}_{v_{1}}}{\partial u}=\left[3, v_{1}\right]\left[2, \frac{\pi}{2}\right][1, \quad \pi]\left[2,-\frac{z_{1}}{z_{N}} v_{1}\right][3,-\varphi] \frac{\partial \boldsymbol{x}}{\partial u} \\
& \underset{1 \varphi}{\boldsymbol{x}}=\frac{\partial \underset{1}{\boldsymbol{x}}}{\partial \varphi}=-\left[3, v_{1}\right]\left[2, \frac{\pi}{2}\right][1, \quad \pi]\left[2,-\frac{z_{1}}{z_{N}} v_{1}\right][3,-\varphi][3] \underset{z}{\boldsymbol{x}}
\end{aligned}
$$

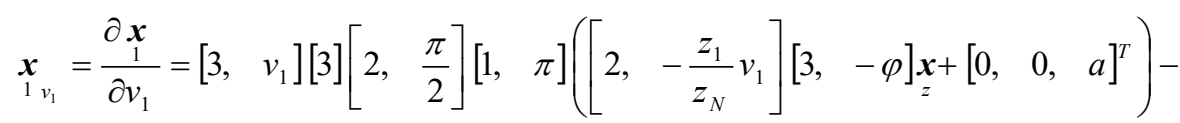

$$
\begin{aligned}
& -\frac{z_{1}}{z_{N}}\left[3, v_{1}\right]\left[2, \frac{\pi}{2}\right][1, \pi]\left[2,-\frac{z_{1}}{z_{N}} v_{1}\right][2][3,-\varphi] \underset{z}{\boldsymbol{x}}
\end{aligned}
$$

On each of the product vectors, a common matrix of rotation acts

$$
m=\left[3, v_{1}\right]\left[2, \frac{\pi}{2}\right][1, \pi]\left[2, \quad-\frac{z_{1}}{z_{N}} v_{1}\right][3,-\varphi]
$$

which can be omitted in the triple product of three vectors.

Therefore, the envelope condition will assume the following form

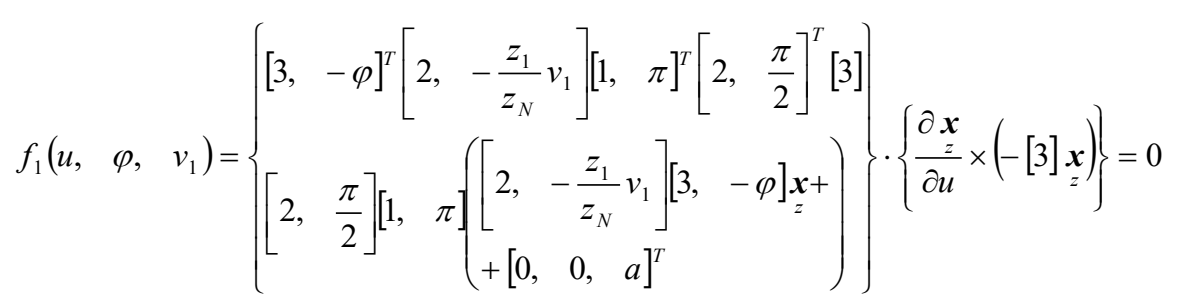


Equation (28) relates three parameters, so it differs significantly from the envelope condition for the case of shaping the cylindrical helical surface, where only two parameters occur (there is no relative tool and worm motion parameter in the envelope condition). The cylindrical helical motion can be written with the helical motion of the characteristic (as well as its arbitrary section) $[13,14]$.

In the case of the globoid helical surface, where the characteristic varies over the worm length, the situation is diametrically different. This is important to the machining of the globoid worm by the hobbing method, whose surface cannot be described with respective motion (i.e. helical motion around the worm axis and rotational motion around the wormwheel axis). This means that the surface of such a worm cannot be made by turning machining (for example, when making a new worm).

\subsection{Globoid worm surface}

A worm surface which is shaped as the envelope of a family of tool action surfaces, that is, which can be described with the system of equations (22) and (28).

$$
\begin{aligned}
& \underset{1}{\boldsymbol{x}}\left(u, \varphi, \quad v_{1}\right)=\left[3, v_{1}\right]\left[2, \frac{\pi}{2}\right][1, \quad \pi]\left(\left[2,-v_{N}\right][3,-\varphi] \underset{z}{\boldsymbol{x}}(u)+\left[\begin{array}{lll}
0, & 0, & a
\end{array}\right]^{T}\right)
\end{aligned}
$$

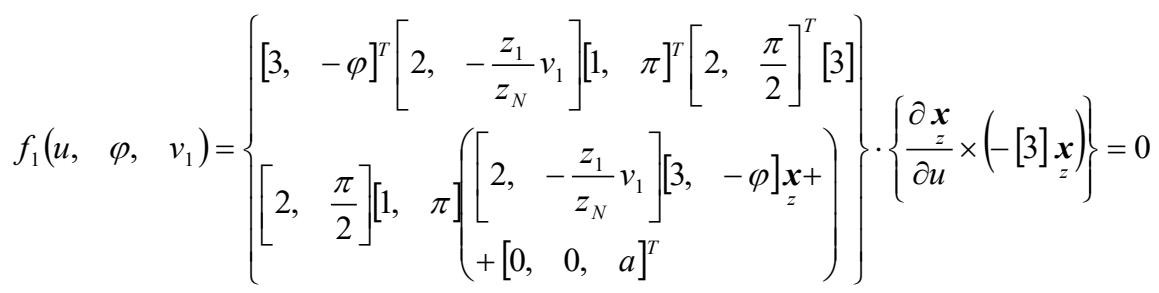

To determine the worm axial profile, the axial section relationship should be additionally taken into account

$$
f_{2}=\underset{1}{x^{2}}\left(u, \varphi, \quad v_{1}\right)=0
$$

that is, for instance, for successively preset values of parameter $\mathrm{u}$, the values of $\varphi$ and $v_{1}$ (and $v_{N}$ ) need to be determined from the system of equations (29b), which, after substituting in equation (29a), enable the determination of the worm profile coordinates.

The worm surface can be defined in a discrete form of points in successive planes parallel to the axial plane; therefore, equation (30) can be substituted with equation

$$
f_{2}\left(u, \varphi, \quad v_{1}\right)=x_{1}^{2}-S=0
$$

where: $S$ - cutting plane position parameter.

For $S=0$, we will determine the profile of the cut by the axial plane).

If we take into consideration a cylindrical worm with one convolution and cut it with the axial plane, then we will see "subsequent teeth" (formed as a result of intersection of that convolution with this plane) with a fixed profile in that section.

In the case of the globoid worm, the situation is diametrically different. In a given cutting plane, the "teeth" have a different profile (the diameter changes, and the convolution lead angle changes). This is of significant importance to the assembly of the gear, which should be very accurate [15]. 


\section{Conclusions}

Two types of the surface of the globoid worm machined according to two different methods have been described.

The worm axial profile has been taken into consideration and, as the performed analysis has shown, for a worm shaped by the hobbing method this profile is variable over the worm length.

At the same time, for successive points of the worm axial surface, the normals to the worm surface have been determined at those points. This is necessary for the development of a new technology for cutting globoid worms. At the same time, these points are uniformly distributed over the profile height. The relationships describing the surface and the normals to the worm convolution surface are much simpler for turned, i.e. ruled worms.

The variability of the axial profile (over the length and perimeter) of a worm shaped by the hobbing method makes the verification of the accuracy of its execution (profile accuracy) very difficult. In that case, the best practical solution is to verify the mating of the worm and the wormwheel on a special stand for checking the trace of tooth adherence in the gear.

\section{References}

1. T. Nieszporek, R. Gołębski, P. Boral, Shaping the Helical Surface by the Hobbing Method. Procedia Engineering 177, pp. 57-63, https://doi.org/10.1016/j.proeng.2017.02.181 (2017)

2. P. Boral, Determination of the Wormwheel Tooth Surface. Procedia Engineering 177, pp. 57-63 https://doi.org/10.1016/j.proeng.2017.02.183 (2017)

3. P.S. Zak, Globoid drive. National Journal of Machine Building. (Moscow 1962)

4. K. Ochęduszko, Spur gear. Construction. (WNT, Warszawa, 1971)

5. T. Marciniak, Technology of worm gear drive. (Exploitation and Technology Institute - National Publish House, Radom, 2013)

6. K. Ochęduszko, Spur gear. Machining and assembly. (WNT, Warszawa, 1971)

7. T. Marciniak, Cylindrical worm gear drive. (PWN, Warszawa, 2001)

8. Z. Kornberger, Worm gear. (WNT, Warszawa, 1961)

9. T. Nieszporek, Automation of calculation of disc and finger cutter outnline for machining of helical surface. Mechanik Nr 7-8, s. 245-249, (1990)

10. F.L. Litvin, Gear Geometry and Applied Theory. (Prentice Hall, New Jersey 1994)

11. F.L. Litvin, Development of Gear Technology and Theory of gearing. NASA RP1406, (1998)

12. T. Nieszporek, Numerical analysis algorithm of worm gear drive. Conference, Present methods of construction and technology of spur gear. p. 127-134. (Łódź 1997)

13. T. Nieszporek, P. Boral, Examination of the Cylindrical Worm Profile. MATEC Web of Conferences 94, 07007, https://doi.org/10.1051/matecconf/20179407007 (2017)

14. P. Boral, Technological determinants of the teething geometry of worm gears with either a fixed or variable pitch worm. Czestochowa University Press of the Czestochowa University of Technology (2018).

15. T. Nieszporek, R. Gołębski, L. Soos, Analysis of the wormwheel toothing accuracy. Technical Gazette, 24(4), 993-1000 (2017) 